\title{
Unusual Event Recognition for Mobile Alarm System
}

\author{
Sooyeong Kwak, Guntae Bae, Kilcheon Kim, and Hyeran Byun \\ Dept. of Computer Science, Yonsei University, Seoul, Korea, 120-749 \\ \{ksy2177, gtbae, kimkch\}@cs.yonsei.ac.kr, hrbyun@yonsei.ac.kr
}

\begin{abstract}
This paper proposes an unusual event recognition algorithm, which is a part of a mobile alarm system. Our systems focus on unusual event. When the system detects the unusual event, the photos of emergency situation are passed to the user's portable devices such as mobile phone or PDA along with event description to help the user's final decision. The system combines the foreground segmentation, object tracking and unusual event recognition to detect the Drop off, Abandon and Steal bag event. The event recognition module constructs the Bayesian network of each event and uses inference algorithm to detect the unusual event. The proposed system tested in PETS2006 and CAVIAR dataset. The proposed algorithm showed good results on the real world environment and also worked at real time speed.
\end{abstract}

Keywords: Mobile alarm system, Background subtraction, Object tracking, Event recognition.

\section{Introduction}

Visual surveillance is a major research area in computer vision. The recent rapid increase in the number of surveillance cameras has led to a strong demand for automatic methods of processing their outputs. Due to this fact, the necessity of automatic techniques which process and analyze human behaviors and activities is more evident each day.

There have been a number of famous visual surveillances systems. The IBM Smart Surveillance System[1] is a middleware offering for use in surveillance systems and provides video based behavioral analysis capabilities. W4[2] system employs a combination of shape analysis and tracking, and constructs model of people's appearances in order to detect and track groups of people as well as monitor their behaviors even in the presence of occlusion and in outdoor environment. The VSAM system[3] can monitor activities over a large area using multiple cameras that are connected into a network. It can detect and track multiple persons and vehicles within cluttered scenes and monitor their activities over long periods of time.

This paper proposes an unusual event recognition algorithm, which is a part of a mobile alarm system. Unlike most previous surveillance system, our systems focus on unusual event. When the system detects the unusual event, the photos of emergency situation are passed to the user's portable devices such as mobile phone or PDA along with event description to help the user's final decision. Fig. 1 shows that overall system flow chart of the proposed event recognition for mobile alarm system. 
Our proposed system can be divided into three parts: foreground segmentation module detects the location of person and bag using background subtraction method; object tracking module tracks the detected objects which deal with occlusion of multiple objects; and event recognition module which integrates the tracking results in order to recognize three unusual events.

This rest of this paper is organized as follows. Video is segmented into background and foreground regions by a background subtraction algorithm described in section 2 . Section 3 presents tracking algorithm. Section 4 describes the event recognition method. Some experimental results are given in Section 5 and then summary and conclusions are presented in Section 6.

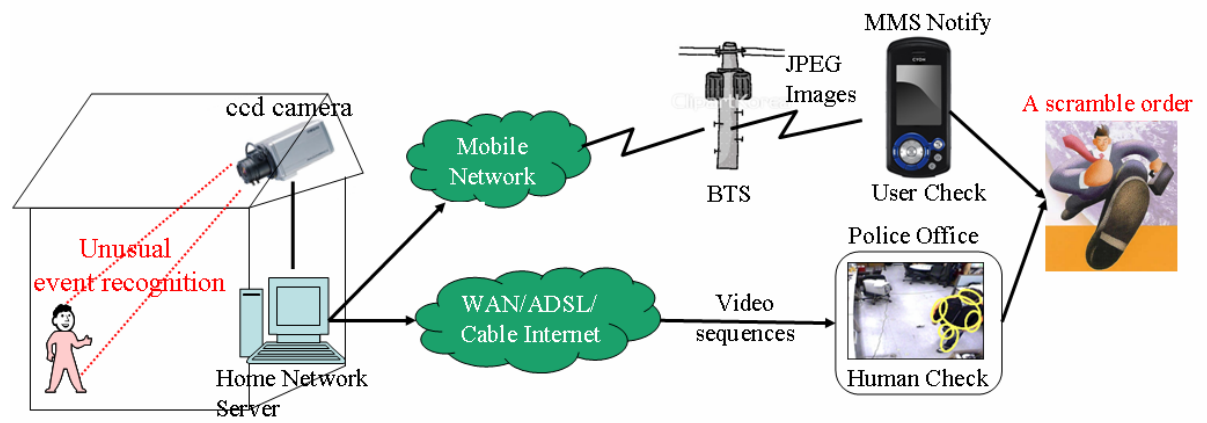

Fig. 1. Overall system flow chart of the proposed event recognition for mobile alarm system

\section{Foreground Segmentation}

In order to extract the foreground region, we used the background subtraction method. Background subtraction has been widely used to detect and track moving object obtained from a static camera. We proposed modified Horprasert's algorithm which call the sequential Horprasert's algorithm.

Horprasert et al.[4] proposed the statistical background model that separates the brightness from the chromaticity component in the batch mode. The batch mode requires long training time and the training images are to be stored in memory while the sequential model does not. Furthermore, the sequential algorithm is of great advantage in practice, especially when cameras can move around and stop to detect foreground objects. We modify the Horprasert's algorithm to work in the sequential mode.

In Horprasert's background model a pixel $p$ is modeled by 4-tuple $<\mu_{p}, \sigma_{p}, a_{p}, b_{p}>$ where is $\mu_{p}$ the expected color value, $\sigma_{p}$ is the standard deviation of RGB color value, $a_{p}$ is the variation of the brightness distortion, and $b_{p}$ is the variation of the chromaticity distortion of pixel $p$. We calculate sequences of $<\mu_{p}{ }^{t}, \sigma_{p}{ }^{t}, a_{p}{ }^{t}, b_{p}{ }^{t}>$ at every $t$ th frame when the scene is stable with the assumption that the sequence approximates $<\mu_{p}, \sigma_{p}, a_{p}, b_{p}>$ as $t$ becomes bigger. It is shown in equation (1). 


$$
<\mu_{p}, \sigma_{p}, a_{p}, b_{p}>\rightarrow<\mu_{p}{ }^{t}, \sigma_{p}{ }^{t}, a_{p}{ }^{t}, b_{p}{ }^{t}>
$$

The sequences are calculated by the following. We define the expectation and standard deviation of the color vector of pixel $p$ as $\mu_{p}{ }^{t}=\left(\mu_{r}{ }^{t}(p), \mu_{g}{ }^{t}(p), \mu_{b}{ }^{t}(p)\right)$ and ${\sigma_{p}}^{t}=\left(\sigma_{r}{ }^{t}(p), \sigma_{g}{ }^{t}(p), \sigma_{b}{ }^{t}(p)\right)$, respectively, up to the $t$ th frame. For sequential background training process we calculate $\mu_{p}{ }^{t}$ and $\sigma_{p}{ }^{t}$ using equation (2) and (3).

$$
\begin{gathered}
\mu_{i}^{t}(p)=\frac{t-1}{t} \mu_{i}^{t-1}(p)+\frac{1}{t} C_{i}^{t}(p) \\
\sigma_{i}^{t}(p)^{2}=\frac{t-1}{t} \sigma_{i}^{t-1}(p)+\frac{1}{t}\left(C_{i}^{t}(p)-\mu_{i}^{t}(p)\right)^{2}
\end{gathered}
$$

Where $i=r, g, b$ and $C_{p}{ }^{t}=\left(C_{r}{ }^{t}(p), C_{g}{ }^{t}(p), C_{b}{ }^{t}(p)\right)$ is the observed color of pixel $p$ at the $t$ th frame. The brightness and chromaticity distortions can be obtained using the temporal mean $\mu_{p}{ }^{t}$ and standard deviation $\sigma_{p}{ }^{t}$. The variation of the brightness distortion $a_{p}{ }^{t}$ and the chromaticity distortion $b_{p}{ }^{t}$ are calculated by equation (4).

$$
\left(a_{p}^{t}\right)^{2}=\frac{t-1}{t}\left(a_{p}^{t-1}\right)^{2}+\frac{1}{t}\left(a_{p}^{t}-1\right)^{2},\left(b_{p}^{t}\right)^{2}=\frac{t-1}{t}\left(b_{p}^{t-1}\right)^{2}+\frac{1}{t}\left(\gamma_{p}^{t}\right)^{2}
$$

The details of the algorithms can be found in [5]. Fig 2 shows results of proposed background subtraction.

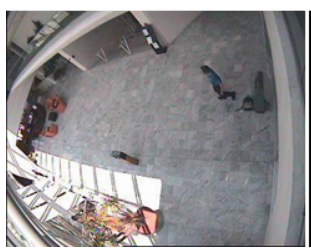

(a)

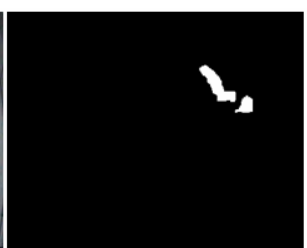

(b)

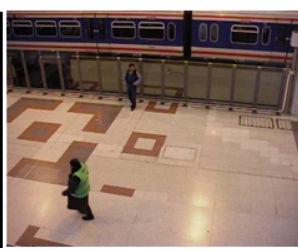

(c)

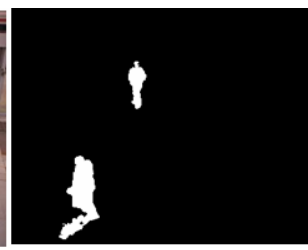

(d)

Fig. 2. The results of Background subtraction (a) Original image in CAVIAR dataset (b) Results of Background subtraction in CAVIAR dataset (c) Original image in PETS2006 dataset (d) Results of Background subtraction in PETS2006 dataset

\section{Object Tracking}

For detected object tracking, we take the results of foreground segmentation which is described by a bounding box (It can be know the location and size of the tracking object). The tracking module applies Senior's method[6] to handle the occlusion and merge/split between multiple object using appearance model. This model is used to localize object during partial occlusions, detect complete occlusions and resolve depth ordering of objects during occlusions. 
At first, the bounding box distance between each of the foreground region and all the currently active tracks is computed to track correspondence. If the distance is lower than the threshold value, we consider that each foreground region associated to tracks. And then, it analyzes four possible results such as existing object, new object, merge detected and split detected. Each case has the predefined rules which are well described in Senior paper[6]. Fig. 3 shows that the processes of tracking when multiple objects are occluded in PETS2006 Dataset.

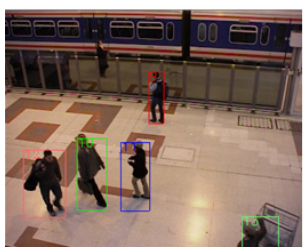

1053

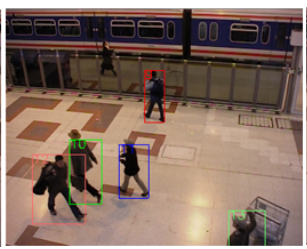

1057

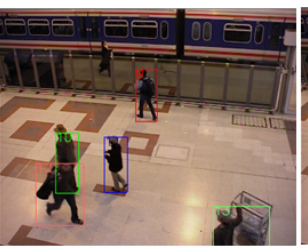

1061

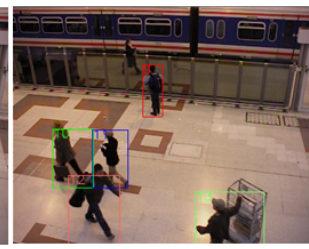

1070

Fig. 3. The processes of tracking when multiple objects are occluded in PETS2006 Dataset, with frame numbers

\section{Event Recognition}

We define three events such as Drop off, Abandon, and Steal bag. In order to recognize these events, we construct Bayesian network in each event which shown in Fig. 4. Our event structure refers to [7]. Drop off bag and Steal bag events need three pieces of evidence and Abandon bag event needs two pieces of evidence. All evidences of each event are described in Table 1. Useful information of tracking object such as speed, direction and the distance of between two object can computed from the bounding box and it used as evidence. If all evidences are observed, we can know the probability of each event using Bayesian inference[8]. Given the evidence, the posterior probability is computed using the equation (5).

$$
P\left(\text { Event } \mid e_{k}^{1}, e_{k}^{2}, \ldots, e_{k}^{n}\right)=\frac{\prod_{i=1}^{n} P\left(e_{k}^{i} \mid \text { Event }\right) P(\text { Event })}{\prod_{i=1}^{n} P\left(e_{k}^{i} \mid \text { Event }\right) P(\text { Event })+\prod_{i=1}^{n} P\left(e_{k}^{i} \mid \neg \text { Event }\right) P(\neg \text { Event })}
$$

where $\{$ Dropp off, Abandon, Steal $\} \in$ Event, $\{D, A, S\} \in k, i=1,2, \ldots, n$

To compute equation (5), several prior and conditional probabilities are needed. The prior and the conditional probability can be determined by expert. These are shown in equation (6). When the posterior probability of each event is over the 0.5 , the alarm is triggered.

$$
P(\text { Event })=0.5, P(\neg \text { Event })=0.5, P\left(e_{k}^{i} \mid \text { Event }\right)=0.9, P\left(e_{k}^{i} \mid \neg \text { Event }\right)=0.5
$$




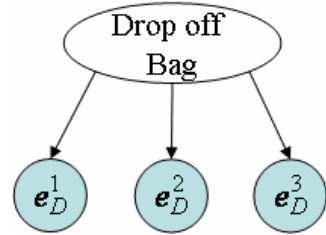

(a)

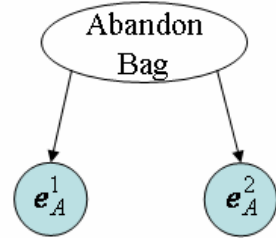

(b)

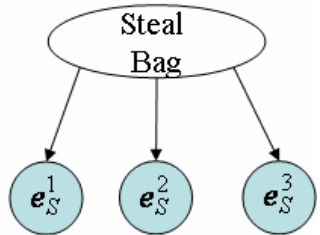

(c)

Fig. 4. The structure of Bayesian networks (a) Bayesian model of Drop-off bag event (b) Bayesian model of Abandon bag event (c) Bayesian model of Steal bag event

Table 1. The definitions of evidence

\begin{tabular}{cl}
\hline Event & Evidence \\
\hline Drop off bag & $e_{D}^{1}:$ The bag did not appear 0.1 second ago. \\
& $e_{D}^{2}:$ The bag shows up now. \\
& less than 30 pixels \\
& $e_{A}^{1}:$ The distance between the person and the bag 0.1 sec- \\
ond ago is less than 40 pixels & $e_{A}^{2}:$ The distance now is larger than 60 pixels \\
& $e_{S}^{1}:$ The person approaches the bag and the distance be- \\
tween the person and the bag is less than 60 pixels. \\
$e_{S}^{2}:$ The person stops near the bag. \\
$e_{S}^{3}:$ The person takes away the bag
\end{tabular}

\section{Experimental Results}

The proposed system was implemented in $\mathrm{C} / \mathrm{C}++$ and was run on Pentium IV-3.0 GHz PC with 1G RAM. We used the PETS2006 Dataset S1 (Take 1-C)[9] and CAVIAR Test Dataset (Person leaving bag by wall)[10] in order to detect the three unusual event, such as the Drop off, Abandon, and Steal bag, in a real world environment. The description of test videos shows below. The average running time of the proposed system was about $13 \mathrm{fps}$.

PETS2006 Dataset S1 (Take 1-C): The scenarios are filmed from multiple cameras and involve multiple actors. The scenario contains a person with a rucksack who loiters before leaving the item of bag unattended. The dataset consists of 1479 frames recording activities in Victoria metro station. The video sequence includes a total of 24 moving objects, people and bag appearing at close, medium and fat distances from the camera. The image size is $360 \times 288$ pixels. 
CAVIAR Test Dataset (Person leaving bag by wall): These include a person leaving a package in a public place. The dataset consists of 837 frames recording activities in the entrance lobby of the INRIA Labs at Grenoble, France. The resolution is halfresolution PAL standard(384x288 pixels, 25 frames per second).

\subsection{Performance of Object Tracking}

Performance of the object tracking was evaluated with respect to the ground truth in each frame of test sequences. The evaluation method refers to [11]. Each frame tested to see if the number of objects as well as their sizes and locations match the corresponding ground truth data for that particular frame. To evaluate the tracking algorithm, we compute True Negative(TN), True Positive(TP), False Negative(FN), and False Positive(FP) for every frame in the sequence. These definitions describe below. Also, we compute the Accuracy using the equation (7).

- TN: Number of frames where both ground truth and system results agree on the absence of any object.

- TP: Number of frames where both ground truth and system results agree on the presence of one or more objects, and the bounding box of at least one or more objects coincides among ground truth and tracker results.

- FN: Number of frames where ground truth contains at least one object, while system either does not contain any object or none of the system's objects fall within the bounding box of any ground truth object.

- FP: Number of frames where system results contain at least one object, while ground truth either does not contain any object or none of the ground truth either does not contain any object or none of the ground truth's objects fall within the bounding box of any system object.

Table 2 shows the performance of the tracking algorithm. Because of the foreground segmentation part does not detect the small moving objects in PETS2006 dataset, the Accuracy is not good.

$$
\text { Accuracy }=\frac{T P+T N}{T F}
$$

where $T F$ is total number of frames.

Table 2. Performance of the tracking algorithms

\begin{tabular}{ccc}
\hline & $\begin{array}{c}\text { PETS2006 Dataset S1 } \\
(\text { Take 1-C) }\end{array}$ & $\begin{array}{c}\text { CAVIAR Test Dataset } \\
\text { (Person leaving bag by wall) }\end{array}$ \\
\hline TP & 1258 & 599 \\
TN & 46 & 195 \\
FP & 45 & 15 \\
FN & 130 & 31 \\
Accuracy & 0.881 & 0.945 \\
\hline
\end{tabular}




\subsection{Performance of Event Recognition}

In order to evaluate the event recognition, we compare the frame number when an even is triggered with our results and the ground truth. The performance of the event recognition shows in Table 3. The key frame of the three events such as Drop off, Abandon and Steal bag shown in Fig. 5. We can see in Table 3, the alarm event is detected within an error of 25 frames except the abandon bag event in PETS2006 Dataset S1. In that sequence, the merge and split algorithm in tracking module has not separate quickly the human and the bag.

Table 3. Performance of event recognition

\begin{tabular}{ccccc}
\hline & \multicolumn{2}{c}{$\begin{array}{c}\text { PETS2006 Dataset S1 } \\
\text { (Take 1-C) }\end{array}$} & \multicolumn{2}{c}{$\begin{array}{c}\text { CAVIAR Test Dataset } \\
\text { (Person leaving bag by wall) }\end{array}$} \\
\cline { 2 - 5 } & Ground truth & Our result & Ground truth & Our result \\
\hline Drop off bag & 1922 & 1935 & 949 & 974 \\
Abandon bag & 2086 & 2100 & 988 & 1003 \\
Steal bag & none & none & 1348 & 1359 \\
\hline
\end{tabular}

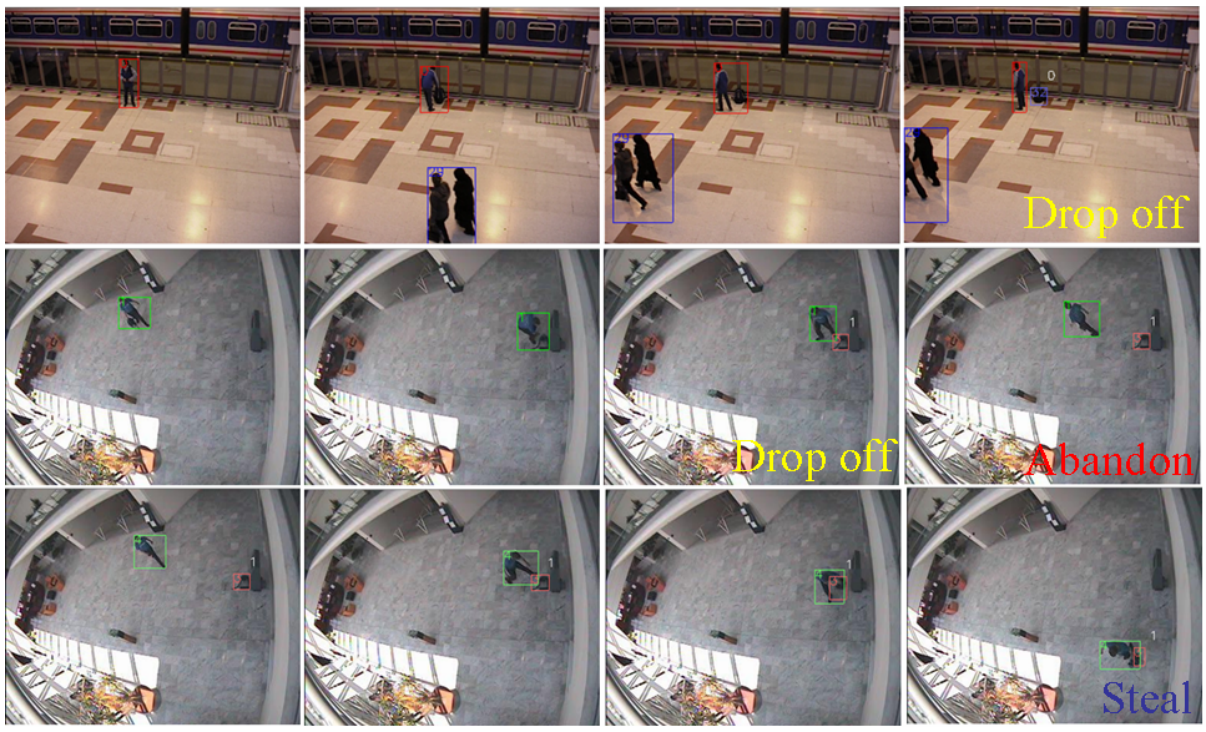

Fig. 5. It shows the key frame of the three events. First row shows the Drop off bag event in PETS2006 test dataset and the second row shows the Drop off bag and Abandon bag event in CAVIAR test dataset. The Steal bag event in CAVIAR test dataset is shown at last row.

\section{Summary and Conclusions}

This paper proposed unusual event recognition for mobile alarm system. Our system has three main modules such as moving object detection, tracking and event recognition. In 
order to detect the foreground object, we used the background subtraction method. After candidate foreground region are detected, appearance model is used for moving object tracking. In this paper, we also used Bayesian inference algorithm in order to recognize the unusual event. The proposed algorithm showed good results on the real world environment and also worked at real time speed. The proposed framework can be easily employed or integrated into a variety of vision surveillance systems.

Acknowledgments. This research was supported by the Ministry of Information and Communication, Korea under the Information Technology Research Center support program supervised by the Institute of Information Technology Assessment, IITA2005-(C1090-0501-0019).

\section{References}

1. Chiao-Fe Shu, Hampapur A., Lu M., Brown L., Connell J., Senior A., and Yingli Tian : IBM smart surveillance system (S3): a open and extensible framework for event based surveillance, IEEE Conference on Advanced Video and Signal Based Surveillance (2005) 318-323

2. I. Haritaoglu, D. Harwood, and L. S. Davis : W : Real-time surveillance of people and their activities, IEEE Transaction on Pattern Analysis and Machine Intelligence, (2000) Vol. 22, 809-830

3. R. T. Collins, A. J. Lipton, T. Kanade, H. Fujiyoshi, D. Duggins, Y. Tsin, D. Tolliver, N. Enomoto, O. Hasegawa, P. Burt, and L.Wixson : A system for video surveillance and monitoring, Carnegie Mellon University, Pittsburgh, PA, Technical Report, CMU-RI-TR$00-12,(2000)$

4. T. Horprasert, D. Harwood, L.S. Davis: A statistical approach for real-time robust background subtraction and shadow detection. Proceeding of IEEE Frame RateWorkshop (1999) 1-19.

5. Jung-Ho Ahn and Hyeran Byun: Human silhouette extraction method using region based background subtraction, International Conference on Mirage 2007 (To be appear)

6. Andrew Senior: Tracking people with probabilistic appearance models, Proceedings 5th IEEE International Workshop on PETS, (2002)

7. Fengjun Lv, Xuefeng Song, Bo Wu, Vivek Kumar Singh, and Ramakant Nevatia.: LeftLuggage Detection using Bayesian Inference, Proceedings $9^{\text {th }}$ IEEE International Workshop on PETS, (2006) 83-90

8. D'Ambrosio : Inference in Bayesian networks, AI Magazine (1999) 21-35

9. http://homepages.inf.ed.ac.uk/rbf/CAVIARDATA1/

10. http://www.cvg.rdg.ac.uk/PETS2006/index.html

11. Faisal Bashir and Fatih Porikli : Performance Evaluation of Object Detection and Tracking Systems, Proceedings $9^{\text {th }}$ IEEE International Workshop on PETS, (2006) 7-13 